\title{
Epidemiology of Sudden Unexpected Death in Infancy: Evidence from the London Metropolitan Police Project Indigo Investigation
}

\author{
Mage $\mathrm{DT}^{* 1}$ and Donner $\mathrm{EM}^{2}$ \\ ${ }^{1}$ Environmental Health Division, World Health Organization, Geneva, CH (retired), Newark, DE, USA \\ ${ }^{2}$ DuPont Haskell Global Centers for Health and Environmental Science, Newark, DE 19711, USA
}

*Corresponding author: Mage DT, Environmental Health Division, World Health Organization, Geneva, CH (retired), 18 W. Periwinkle Ln, Newark, DE 19711, USA, E-mail: magedonner@aol.com

Citation: Mage DT, Donner EM (2017) Epidemiology of Sudden Unexpected Death in Infancy: Evidence from the London Metropolitan Police Project Indigo Investigation. J Paediatr Neonatal Dis 2(1): 101. doi: 10.15744/2456-5482.2.101

\begin{abstract}
Background: The London Metropolitan Police in collaboration with medical personnel, study all sudden unexpected death in infancy (SUDI) $<2$ years in their jurisdiction, to identify suspicious cases for further investigation. The 2005-2010 Project Indigo includes extensive data on all such non-suspicious cases.

Methods: Deidentified data on age and gender of 477 infants dying a natural unexpected-sudden death in London were gathered for statistical analyses, for comparison to our published a priori probability models that predict their distributions without need of superfluous information, such as race, autopsy findings, or SUDI risk factors.

Results: The total observed male fraction of 0.5639 for all these 477 Indigo cases ( 269 male) is predicted using a recessive X-linkage model for Sudden Infant Death Syndrome (SIDS) as 0.5676. The transformed age distribution of all 477 Indigo cases of different causes of death is modeled by a single four-parameter lognormal distribution, $y=\log [(d+9.44) /(1254-d)]=\mu+\sigma z$, where $d$ is Indigo age in calendar days of life $(d=\mathrm{DOD}-\mathrm{DOB} \geq 0)$, median $\mu=-1.085$, slope $\sigma=0.543$, and $z$ is a standard normal deviate.

Conclusion: All liveborn infants undergo a similar genetically programmed physiological development and pass through periods at which they are at risk for respiratory and cardiac failure from natural causes related to gender and age that may be correlated with environmental risk factors. The age distribution of all non-surviving infants appears as an integral of probability of simultaneous causal factors such as physiological anemia, fulminating prodromal respiratory infection and absence of a dominant X-linked allele that is protective of cerebral anoxia. This results in a universal 4-parameter lognormal age distribution that by Cramér's Theorem must be the sum of component lognormal distributions of natural deaths from such various respiratory and cardiac causes.

Keywords: Infant death; SIDS; SUDI; Indigo; X-linkage; Cramér's Theorem; Anemia

List of abbreviations: SIDS: Sudden Infant Death Syndrome; SUDI: Sudden Unexpected Death in Infancy; U.K.: United Kingdom; U.S.: United States; CDC: U.S. Centers for Disease Control and Prevention; DOD: Date of Death; DOB: Date of Birth; MPS: London Metropolitan Police Service; ARI: Acute Respiratory Infection
\end{abstract}

\section{Background}

In the absence of fatal trauma and criminally-suspicious circumstances (evidence of possible infanticide or neglect), infants die from natural causes by either respiratory failure (breathing stops first) or cardiac failure (heart stops beating first). We neglect cases, such as fall from great height, where both stop simultaneously. We have shown previously using autopsied Sudden Infant Death Syndrome (SIDS) as a model, that infant deaths by natural respiratory causes have a $\sim 50 \%$ male excess and a $\sim 0 \%$ male excess from natural cardiac causes, for equal numbers of XY male and XX female infants at risk [1-5].

Table 1 shows the U.S. male fractions 1968-2015 from five widely different respiratory causes of infant death all having almost the same male fraction of order 0.60 [6]. Note the male fraction of accidental inhalation of food, foreign object, and gastric content (0.5963) is virtually identical to 0.5999 for SUDI. Given that XY male and XX female infants are at equal risk of such inhalation accident, more females than males have an inherent ability to survive the anoxic crisis that can only come from their extra X-chromosome [7]. (We are not able to correct for possible XXY male and XXX female gender, as those data are not within the Indigo data base). 


\begin{tabular}{|c|c|c|c|}
\hline $\begin{array}{c}\text { a. SUDI = } \\
\text { SIDS+UNK+ASSB }\end{array}$ & PNN Male 28-364 days & $\begin{array}{l}\text { PNN Female 28-364 } \\
\text { days }\end{array}$ & Male Faction \\
\hline $\begin{array}{c}\text { 1999-2015 R95, R99, } \\
\text { W75 }\end{array}$ & 33,370 & 22,838 & 0.5937 \\
\hline $\begin{array}{c}\text { 1979-1998 798, 799.9 } \\
\text { E913.0 }\end{array}$ & 61,783 & 40,276 & 0.6054 \\
\hline $1968-1978$ 795, 796.0 & 17,794 & 12,229 & 0.5927 \\
\hline 1968-2015 Total & 112,947 & 75,344 & 0.5999 \\
\hline $\begin{array}{l}\text { b. Anomalies of the } \\
\text { great vessels }\end{array}$ & Male $<5$ years & Female $<5$ years & Male Fraction \\
\hline 1999-2015 Q26 & 419 & 276 & 0.6029 \\
\hline $1979-1998747.9$ & 693 & 470 & 0.5959 \\
\hline 1968-1978 747.4 & 1,023 & 605 & 0.6284 \\
\hline 1968-2015 Total & 2,135 & 1,351 & 0.6124 \\
\hline $\begin{array}{c}\text { c. Acute Respiratory } \\
\text { Infections, Upper and } \\
\text { Lower ARI }\end{array}$ & Male $<1$ year & Female $<1$ year & Male Fraction \\
\hline 1999-2015 J00-J22 & 2,977 & 2,095 & 0.5869 \\
\hline $1979-1998$ 460-487 & 9,124 & 6,464 & 0.5853 \\
\hline 1968-1978 460-486 & 27,982 & 20,950 & 0.5719 \\
\hline 1968-2015 Total & 40,083 & 29,509 & 0.5760 \\
\hline $\begin{array}{l}\text { d. Respiratory Distress } \\
\text { Syndrome, Hyaline } \\
\text { Membrane disease }\end{array}$ & Male $<1$ year & Female $<1$ year & Male Fraction \\
\hline 1999-2015 P22.0 & 6,680 & 4,604 & 0.5920 \\
\hline 1979-1998 769 & 36,373 & 23,135 & 0.6112 \\
\hline $1968-1978776$ & 99,264 & 66,455 & 0.5990 \\
\hline 1968-2015 Total & 142,217 & 94,194 & 0.6016 \\
\hline $\begin{array}{l}\text { e. Inhalation of food, } \\
\text { foreign object and } \\
\text { gastric content }\end{array}$ & Male $<5$ years & Female $<5$ years & Male Fraction \\
\hline 1999-2015 W78 - W80 & 1,418 & 824 & 0.6325 \\
\hline 1979-1998 E911- E912 & 3,070 & 2,030 & 0.6020 \\
\hline 1968-1978 E911 - E912 & 4,536 & 3,256 & 0.5821 \\
\hline 1968-2015 Total & 9,024 & 6,110 & 0.5963 \\
\hline
\end{tabular}

Grand Total; 306,406 Male, 206,508 Female: 0.5974 male fraction

Table 1: U.S. 1968-2015 CDC infant mortality data from respiratory failures showing a $50 \%$ male excess: a) Sudden Unexpected Death in Infancy (SIDS, Unknown Causes, Accidental Suffocation and Strangulation in Bed); b) Anomalies of the great vessels; c) Acute upper and lower Respiratory Infections; d) Respiratory Distress Syndrome; and e) Inhalation of food, foreign object or gastric content. ICD-8, ICD-9, ICD-10 codes as shown [6]

In addition, we have also shown that the age distribution of infant natural deaths from respiratory causes follow a Johnson $S_{B}$ Distribution (a.k.a. 4-parameter lognormal) [8-11]. In this paper, we use the infant mortality data compiled by the London Metropolitan Police Service (MPS project Indigo) for years 2005-2010 on all 477 non-suspicious Sudden Unexpected Death in Infancy (SUDI) (in this case up to 2-years of age) [12]. In the United Kingdom (U.K.) all SUDI cases of unattended deaths require forensic investigation by expert pediatric pathologists following procedural guidelines. Consequently these 477 SUDI cases constitute a collection of SUDI deaths by both explained and unexplained causes, as well as "unascertained" deaths in which abnormalities were found at autopsy that were not apparently consistent with the cause of the death [12]. The authors make the point that these 477 SUDI cases were autopsied at different London hospitals by different pediatric pathologists who often have varying opinions on cause of death when findings are ambiguous. This may increase the variance of these data.

Whereas, the full Indigo dataset contains over 140 fields for each case involving variables "relating to the circumstances and cause of death, including social and behavioral factors of parents and caregivers" we requested only two of those fields of data for this study of those 477 cases to evaluate our genetic model : 1) The age in number of days at the time of death [born and died on same day $=0$, born and died on following day $=1$, etc.]; and 2) gender of infant as male or female [12]. We showed in our previous work that information on whether the SUDI death was explained or unexplained, or race, ethnicity, or risk factors were included, was not necessary for input to test our genetic based model which is the purpose of this study $[1-5,10]$. 


\section{Methods}

The anonymized Indigo data on age and gender presented here were made available to us by Dr. Neil J. Sebire, the corresponding author of Bamber, et al., and are similarly available to the reader from him on request [12]. In this study, we compare these London Indigo data set results with other published infant mortality data sets from the U.K., Colombia and the U.S., to show: 1) whether the observed male fraction of these data is predicted by our X-linkage model for the male fraction of all infant mortality; and 2) whether the Indigo infant ages display the same type of 4-parameter lognormal age distribution that we have shown for SIDS and SUDI [6,11,13-17]. The median (50 percentile) and slope of the line (geometric standard deviation) are the first and second parameters, respectively. We also used the same third and fourth parameters for Figures 1 and 2 that we reported previously, using the method of maximum likelihood [10].

\section{Results - Indigo Gender and Age Distributions}

Of the 477 Indigo cases, 269 were male and 208 were female, for a male fraction of 0.5639 . We have shown previously that SIDS has a 50\% male excess for equal numbers of XY males and XX females at risk that also holds for all other natural infant deaths by respiratory failure [1-4]. It was first proposed by Naeye, et al. that a recessive X-linkage must be responsible for the male excess infant mortality [7]. We agree and proposed that a non-protective recessive X-linked allele in Hardy-Weinberg Equilibrium with frequency $q=2 / 3$ would place $2 / 3$ of all XY males and $4 / 9$ of all XX females at risk, providing the $50 \%$ male excess [2,3]. We also showed that on the same basis there appears to be no (0\%) male excess mortality from natural causes by cardiac failure [16,17].

Figure 1 shows the age distribution of 475 of these Indigo data (two cases only had gender reported), ranging from 0 to 724 days, plotted as a left-censored 4-parameter lognormal distribution with third parameter equal -9.44 days and fourth parameter 1,254 days, as determined by us as valid estimators for respiratory failures from our previous published analyses of global SIDS data $[10,11]$. Only the main body of the total Indigo age-distribution is well fit by the SIDS model, with under-prediction at the low age end and over-prediction at the high age end. By inspection there appears to be a discontinuity in the slope of the Figure 1 curve at the completion of three days of life, after the four horizontal segments representing 38 neonatal cases $\{7,6,13,12\}$ dying at $0,1,2$ and 3 days of life, respectively. We reason that these neonatal deaths may likely be from "Postnatal Collapse," a different phenomenon than the other Indigo post-neonatal deaths because of the known birth-proximate causes of mortality, such as hyaline membrane disease, antenatal aspiration and infection, major congenital malformations, etc. [18,19]. Figure 2 shows the age distribution of 809 SUDI infant death in Colombia (excluding Bogatá) 2005-2010, where local practice assigned ICD-10 W84 (Other unspecified threats to breathing) rather than SIDS (R95), Unknown causes (R99) and W75 (Accidental suffocation and strangulation in bed) [15]. The same discontinuity in slope at the neonatal lower ages as in Figure 1, indicates that this effect is reproducible.

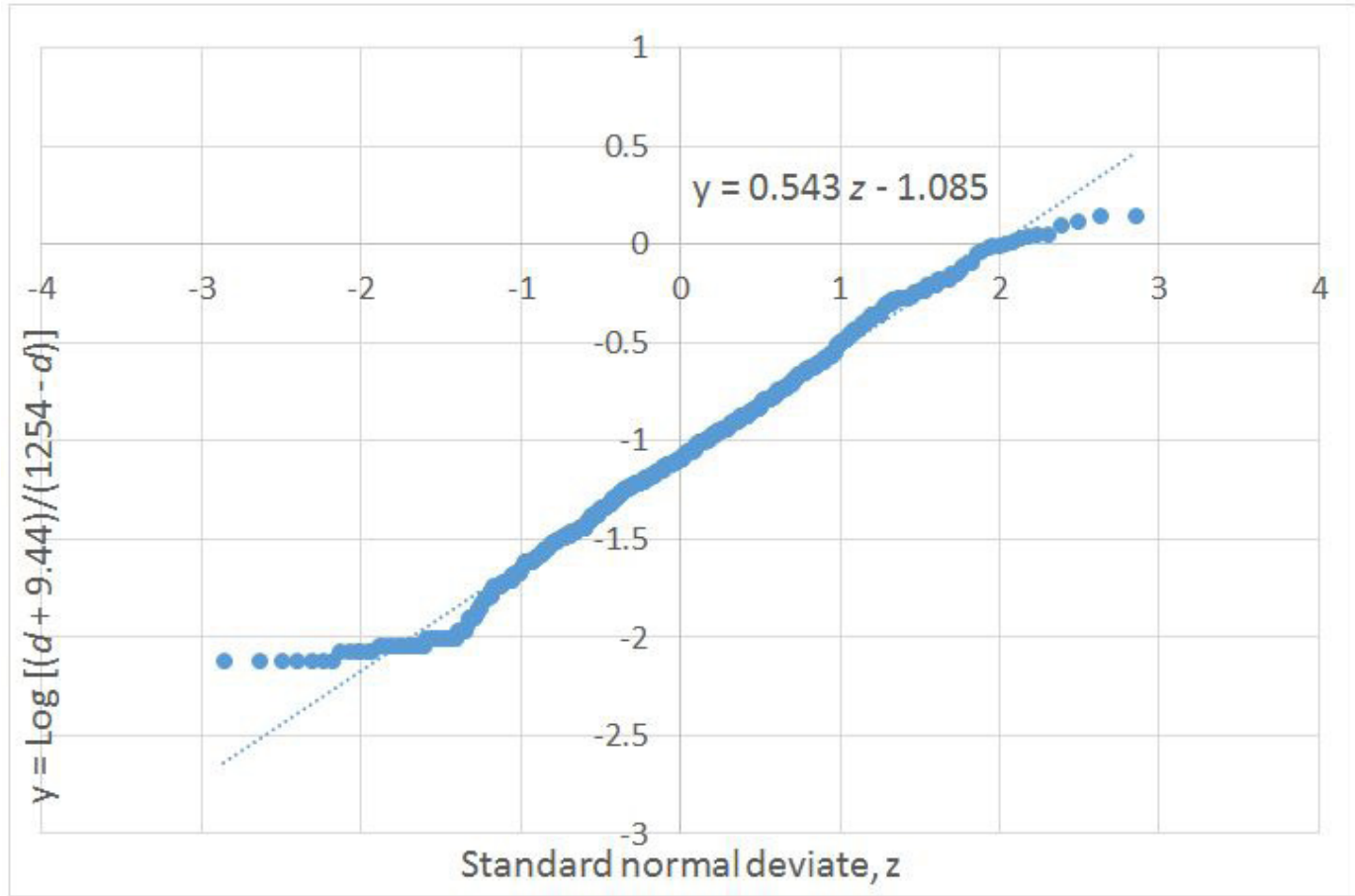

Age in days (d) at death of $\mathrm{N}=475$ Indigo SUDI cases, cardiac and respiratory, plotted as a left-censored 4-parameter lognormal distribution, with $3^{\text {rd }}$ and $4^{\text {th }}$ parameters identical to those found for our previous SIDS age data analyses $[10,11]$. Note discontinuity in slope at $\mathrm{d}=4$, indicating 38 possible cases of 'sudden unexpected postnatal collapse' $[18,19]$

Figure 1: London Indigo age data, $\mathrm{N}=475$ 


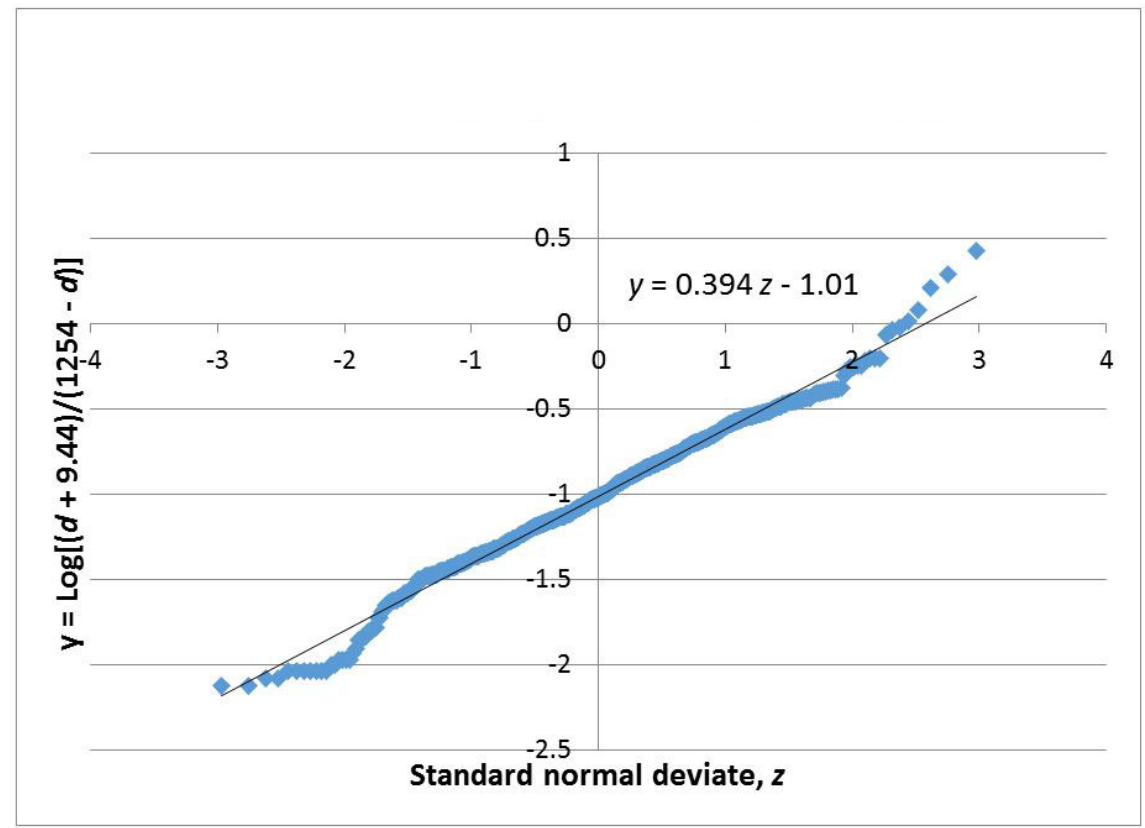

Age in days ( $\mathrm{d}$ ) at death of $\mathrm{N}=809$ SUDI cases in Colombia (excluding Bogatá) 2005 -2010, reported as ICD-10 W84, other unspecified threats to breathing [15]. The third and fourth parameters are the same as for Figure 1. Note the similar discontinuity in slope at neonatal ages as for Figure 1

Figure 2: SUDI W84 (Other unspecified threats to breathing) Colombia excluding Bogotá, 2005-2010, $N=809$ [15]

On this basis, we culled out those 38 neonatal cases cited above and reanalyzed the remaining 437 Indigo cases at ages $d=4$ to 724 days, plotting them by their probability in rank order ( 1 to 437 ) divided by 438, as shown on Figure 3 . For the remaining multiple deaths on a given day after birth, rather than plotting all 5 cases with $\mathrm{DOD}-\mathrm{DOB}=4.0$, we assumed they were uniformly distributed over the $4^{\text {th }}$ day of life as $4.1,4.3,4.5,4.7$ and 4.9 days to make it more realistic.

For the age distribution analysis, we used the graphical procedure of Gibrat as described by Aitchison and Brown [8]. The $3^{\text {rd }}$ and $4^{\text {th }}$ parameters, the lower and upper age bounds, respectively, are adjusted to make the $\mathrm{n}$ ranked ages from 1 to $\mathrm{n}$, plotted as the age

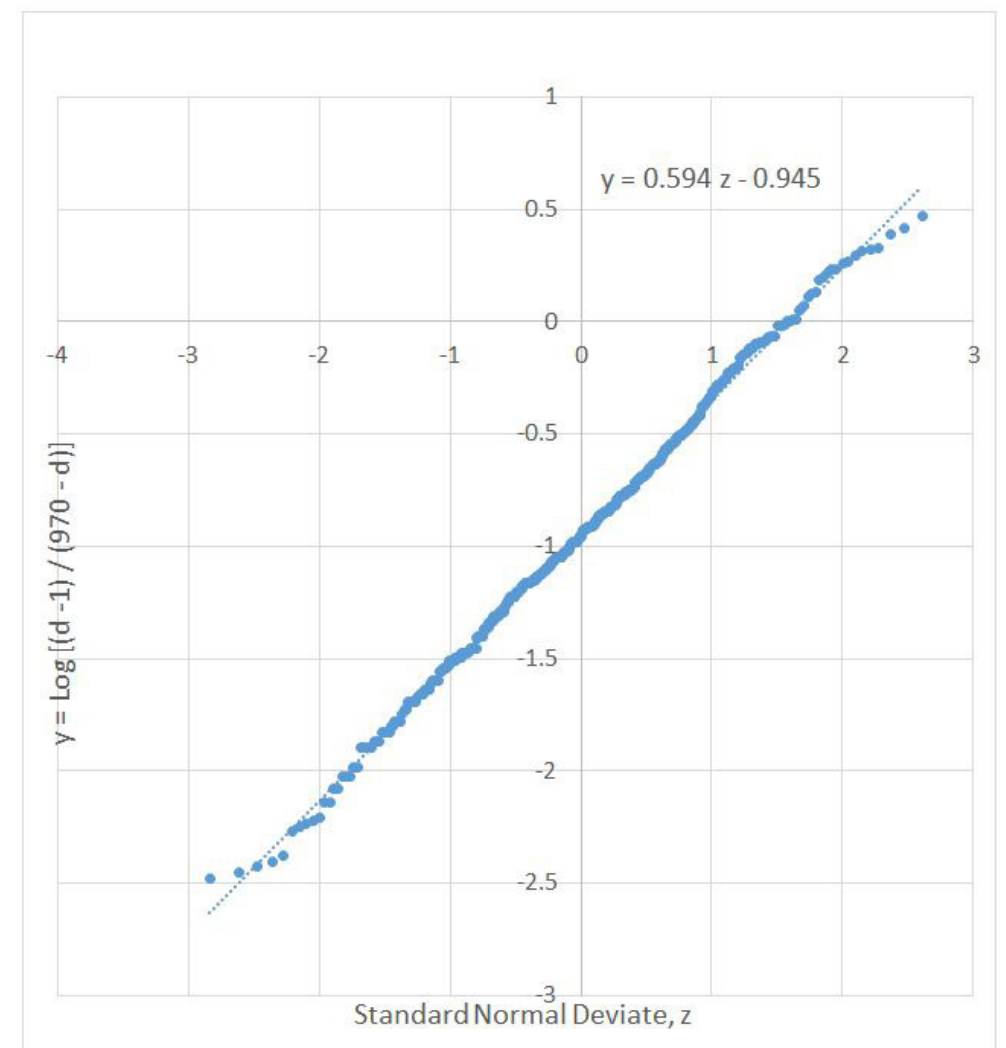

Age in days (d) at death of $\mathrm{N}=437$ Indigo cases excluding 38 cases of possible postnatal collapse with $d<4$. A 4-parameter lognormal distribution with parameters, as $y=\log [(d-1) /(970-d)]$ vs $z$, now fits the entire modified dataset over the range $4 \leq \mathrm{d}<970$, for both cardiac and respiratory failure cases

Figure 3: London Indigo Age Data, $\mathrm{N}=437$ without neonatal collapse 
transform versus $[\mathrm{rank} /(\mathrm{n}+1)]$ converted to standard normal deviates, into a best straight line. Now these 437 data are better fit over their complete range as opposed to Figure 1. Figure 3 shows that the entire truncated (SUDI $>2$ years not included) Indigo post-neonatal data set can be modeled with slight adjustment to the $3^{\text {rd }}$ and $4^{\text {th }}$ parameters, as a single 4 -parameter lognormal distribution.

\section{Discussion}

We reason that if all infants were equally exposed to risks of cardiac and respiratory failure then for each two female cardiac cases there would be two male cardiac cases ( $0 \%$ male excess), and two female respiratory cases. From the observed $50 \%$ male excess respiratory death rate, there would also be three male respiratory deaths. Thus, we predicted that the total infant male excess would simply be their average of $25 \%$, or 5 male deaths per 4 female deaths for a total male fraction of $5 / 9=0.5555$ for equal numbers of male and female infants at risk $[16,17]$. However, there is a male birth rate excess of order $5 \%$, in developed countries where there is no bias against female infant births, which would increase the predicted male fraction from 20 males per 16 females, to 21 males per 16 females resulting in a predicted male fraction as 21 male/37 total $=0.5676$. Without information on the daily average numbers of male and female infants under 2 years at risk living in Greater London during the 2005-2010 study period, we use the nominal $5 \%$ male excess prediction of 0.5676 to compare to the observed 0.5639 Indigo data value, which is almost the same as predicted. Carpenter and Gardner reported on all infant mortality from 1 week up to 1 year in England and Wales, 19691976 [13]. They reported that for all causes of death there were 22,965 males in a total of 40,467 infant deaths, a male fraction of 0.5675, virtually exactly as we predicted for a 5\% male excess at risk. The European Union reported that in the entire U.K., 19932012, with a $4.95 \%$ excess of males at risk under 5 years, there were 49,253 male and 37,994 female infant deaths for a male fraction of 0.5644 [14]. In the U.S., CDC reported that between 1968 and 2015 there were 1,238,170 male, and 944,227 female infant deaths under 5 years, with a $4.62 \%$ male excess at-risk, giving a male fraction of 0.5673 that again is almost exactly equal to our predicted $\left[20^{\star} 1.0462 /\left(16+20^{\star} 1.0462\right)\right]=0.5667[6]$.

We do not propose that these Indigo results above mean that all Indigo cases are independent random probability samples from a single 4-paramete lognormal distribution. Rather, we suggest that Indigo cases of respiratory failure are 4-parameter lognormally distributed as we showed previously, and cases of cardiac failure must be also independently 4-parameter lognormally distributed, albeit with different parameters, as required by Cramér's Theorem [10,11,20,21]. That is, if a variable such as the age of all Indigo cases is lognormally distributed and the Indigo cases are the total of respiratory failure cases and cardiac failure cases, then the ages of these respiratory cases and cardiac cases must also be independently lognormally distributed. This is surprising because each Indigo case is independent of all the others, representing independent random samples from a heterogeneous population with different races, ethnicities, economic status, educations, access to medical care, etc. We have shown previously that what is the uniting commonality amongst all these cases may be the mode of death, either respiratory failure or cardiac failure, that gives only one degree of freedom. The late John Emery first modeled SIDS in 1982 as a death caused by cerebral hypoxia leading to acute anoxic encephalopathy, and diagrammed how this condition could be caused by many combinations of factors [22]. We have adapted Dr. Emery's model (with his permission) to include the hypothesized recessive X-linkage as a 'gate' function to determine whether the transient cerebral anoxic episode goes to completion or can be survived $[11,23]$.

\section{Conclusion}

Because all these Indigo SUID post-neonatal ages are jointly lognormally distributed, and are the sum of respiratory failures and cardiac failures, we propose as a finding that their respiratory and cardiac failure ages must separately be lognormally distributed as required by Cramér's Theorem [20,21]. The single equation of Figure 3 fitting the entire distribution, without the subset of post-natal collapse, indicates that there are no other appreciable Indigo subsets of SIDS involved, such as infanticide by gentle smothering, medullary serotonin neuron abnormalities, or enlarged thymus occluding the trachea or major blood vessels. In summary, the age and gender model fit is excellent and supports a common terminal event of acute anoxic encephalopathy for respiratory control neurons in the brain stem. Should an enzyme associated with a dominant X-linked protective allele $(p=1 / 3)$ be identified, prophylaxis against respiratory failure may be possible. The lognormal distribution of SUID ages also suggests that physiological anemia may explain the peak SIDS rate at or about 60 days. Indeed, infants with sickle cell trait or disease have the highest rates of SIDS [24]. Perhaps delayed cord clamping (placental transfusion), followed by a simple hematocrit measurement after birth, may identify those infants at maximal risk for SUID, who would benefit from medical surveillance and treatment to raise their hemoglobin if indicated.

\section{Authors' contributions}

Mage DT conceived of the study. Donner EM performed the gender analysis; Mage DT performed the age analysis. Both authors helped to draft the manuscript and approved the final draft.

\section{Ethics}

The indigo data set was created with no personal identifiers by the London MPS, and reviewed and cleared for release and public use by the London (Bloomsbury) National Research Ethics Service Committee. 


\section{References}

1. Mage DT, Donner EM (1996) An X-linked genetic susceptibility for SIDS and respiratory failures. J Sudden Infant Death and Infant Mortality 1: 295-306.

2. Mage DT, Donner M (1997) A genetic basis for the sudden infant death syndrome sex ratio. Med Hypotheses 48: 137-42. PMID: 9076995.

3. Mage DT, Donner EM (2004) The fifty percent male excess of infant respiratory mortality. Acta Paediatr 93: 1210-5. PMID: 15384886.

4. Mage DT, Donner EM (2014) Is excess male infant mortality from sudden infant death syndrome and other respiratory diseases X-linked? Acta Paediatr 103: 188-93. PMID: 24164639

5. Mage DT, Donner EM (2016) Comment on Fard et al's Candidate gene variants of the immune system and sudden infant death syndrome. Int J Legal Med 130: 1069-70. PMID: 27188625.

6. Centers for Disease Control and Prevention (2016) National Center for Health Statistics. CDC WONDER online Database, compiled from compressed mortality file CMF 1968-2015, USA.

7. Naeye RL, Burt LS, Wright DL, Blanc WA, Tatter D (1971) Neonatal mortality, the male disadvantage. Pediatrics 48: 902-6. PMID: 5129451.

8. Aitchison J, Brown JAC (1973) The Lognormal Distribution with special reference to its uses in economics, Cambridge University Press, London.

9. Mage DT (1980) An explicit solution for $\mathrm{S}_{\mathrm{B}}$ parameters using four percentile points. Technometrics 22: 247-51.

10. Mage DT (1996) A probability model for the age distribution of SIDS. J Sudden Infant Death Syndrome and Infant Mortality 1: 13-31.

11. Mage DT, Donner EM, Vennemann M, Fleming P, Sol-Church K, et al. (2012) All sudden infant respiratory deaths may result from the same underlying mechanism. Scand J Forensic Sci 18: 2-10.

12. Bamber AR, Kiho L, Upton S, Orchard M, Sebire NJ (2016) Social and behavioural factors in Non-suspicious unexpected death in infancy; experience from metropolitan police project indigo investigation. BMC Pediatr 16: 6, doi: 10.1186/s12887-016-0541-x. PMID: 26759055.

13. Carpenter RG, Gardner A (1982) Variations in unexpected infant death rates relating to age, sex, and season In: Studies on medical and population subjects No. 45. HMSO, London, U.K.

14. European Commission, Eurostat. http://epp.eurostat.ec.europa.eu/ portal/page/portal/statistics/themes. Accessed July 31 , 2014.

15. Latorre ML, Barbosa S, Hernández LJ, Mage D (2015) Sudden infant death syndrome and epidemiology of sudden and unexpected infant deaths in Colombia 2005 and 2010 Pediatr 48: 9-14 (Spanish).

16. Mage DT (2012) Do infants die of sudden infant death syndrome (SIDS) with long QT syndrome (LQTS) or from LQTS? Pediatr Cardiol 33: 1472. PMID: 22868671

17. Mage DT, Donner EM (2015) An explanation of the 25\% male excess mortality for all children under 5. Scand J Forensic Sci 21: 2-5.

18. Herlenius E, Kuhn P (2013) Sudden unexpected postnatal collapse of newborn infants: a review of cases, definitions, risks, and preventive measures. Transl Stroke Res 4: 236-47. PMID: 23518795.

19. Well Child (2011) Guidelines for the investigation of newborn infants who suffer a sudden and unexpected postnatal collapse in the first week of life. Gloucestershire, UK.

20. Mage DT, Donner M (2010) Cramér's Theorem proves that SIDS is a distinct entity, and not a collection of different causes of death. J Paediatrics and Child Health 46 (Supp 3): 15.

21. Weisstein, Eric W (2003) Cramér's Theorem From MathWorld--A Wolfram Web Resource. http://mathworld.wolfram.com/CramersTheorem.html

22. Emery JL. A way of looking at the possible causes of crib death. in Sudden Infant Death Syndrome. Eds. Tildon JT, Roeder LM, Steinschneider A. Academic Press, New York, 1983. 123-32.

23. Mage DT, Donner EM (2011) The universal age distribution of the Sudden Infant Death Syndrome. Scand J Forensic Sci 17: 7-10.

24. Vix J, Buguet A, Straboni S, Beidari H (1987) Sudden infant death and sickle cell anemia in the Sahel region of Africa. Med Trop (Mars) 47: 153-9. PMID: 3626817 (French). 